University of Arkansas, Fayetteville

ScholarWorks@UARK

Graduate Theses and Dissertations

$5-2014$

Psychometric Evaluation of a Standardized Set of Alcohol Cue Photographs to Assess Craving

David Edison Lovett

University of Arkansas, Fayetteville

Follow this and additional works at: https://scholarworks.uark.edu/etd

Part of the Applied Behavior Analysis Commons, Clinical Psychology Commons, and the Psychiatric and Mental Health Commons

\title{
Citation
}

Lovett, D. E. (2014). Psychometric Evaluation of a Standardized Set of Alcohol Cue Photographs to Assess Craving. Graduate Theses and Dissertations Retrieved from https://scholarworks.uark.edu/etd/ 2350

This Thesis is brought to you for free and open access by ScholarWorks@UARK. It has been accepted for inclusion in Graduate Theses and Dissertations by an authorized administrator of ScholarWorks@UARK. For more information, please contact scholar@uark.edu. 
Psychometric Evaluation of a Standardized Set of Alcohol Cue Photographs to Assess Craving 


\section{Psychometric Evaluation of a Standardized Set of Alcohol Cue Photographs to Assess Craving}

\section{A thesis submitted in partial fulfillment of the requirements for the degree of Master of Arts in Psychology}

by

David Edison Lovett Oklahoma State University

Bachelor of Science in Psychology, 2010

May 2014

University of Arkansas

This thesis is approved for recommendation to the Graduate Council.

Dr. Lindsay Ham

Thesis Director

Dr. Bill Levine

Dr. Jennifer Veilleux

Committee Member

Committee Member 


\begin{abstract}
To date, research paradigms using alcohol-related stimuli have been limited in their reliability and generalizability due to a lack of published studies examining the psychometric properties of alcohol cues. The primary aim of the current study was to examine the factor structure of a set of alcohol cues and the associated validity and reliability of examining craving for alcohol in an alcohol cue-reactivity paradigm. Participants $(N=195,74.4 \%$ male, $56.4 \%$ Asian, Asian American, or Pacific Islander) completed a web-based survey in which valence, arousal, and craving ratings were obtained following presentation of picture cues. Prior to picture cues, participants completed measures to assess convergent and discriminant validity. Principal component analysis indicated that ratings of alcohol craving following the alcohol cues formed one factor including all 60 cue-elicited craving ratings. Results also supported convergent validity as craving for alcohol following alcohol cue photos were positively correlated with measures of general craving, self-reported past year hazardous alcohol use, and fun-seeking behavior and behavioral drive. Craving for alcohol was found to be significantly higher following alcohol cues compared to neutral cues and unrelated to behavioral inhibition and severity of nicotine dependence, supporting discriminant validity. These findings, taken as a whole, provide support that the set of alcohol cues created in the current study are a reliable and valid set of alcohol cues for the use in alcohol cue reactivity paradigms. Future studies may benefit from assessing reactivity to alcohol cues using this set of validated photographic cues as it may facilitate a greater understanding of the affective processes associated with alcohol use and allow for more targeted behavioral change interventions for alcohol-related problems.
\end{abstract} Keywords: alcohol, craving, subjective reactivity, cue, photographs 
(C2014 by David Edison Lovett All Rights Reserved 


\section{ACKNOWLEDGEMENTS}

I would like to express my sincere gratitude to my advisor Dr. Lindsay Ham for her continued guidance, patience, motivation, and enthusiasm. Special thanks are also due my thesis committee members Dr. Jennifer Veilleux and Dr. Bill Levine for their invaluable encouragements and patience along the way. Further, I would like to thank my fellow lab mates at the Laboratory for Anxiety and Substance Abuse Research (LASAR) at the University of Arkansas for their collaborative spirit, friendly competition, and overall support of research endeavors. 


\section{TABLE OF CONTENTS}

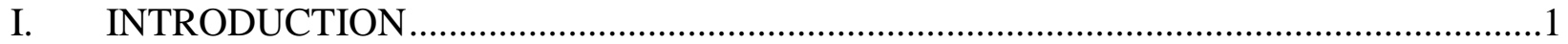

A. ALCOHOL CUE-INDUCED CRAVING THEORY ………………................................

B. ALCOHOL CUE-INDUCED CRAVING METHODS ....................................................

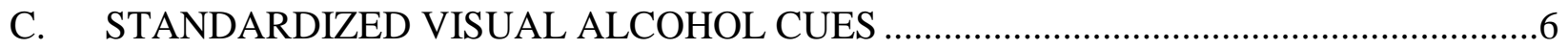

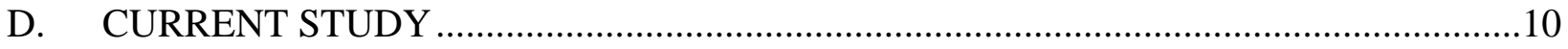

Psychometric Evaluation of Alcohol Cues ……………...................................................

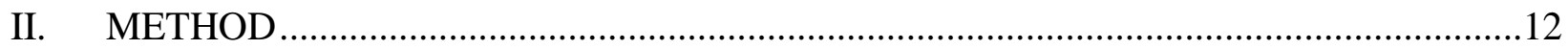

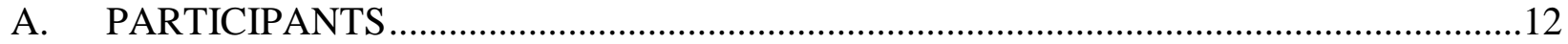

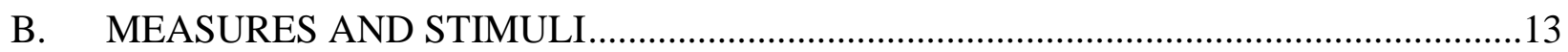

Self-report Questionaires...................................................................................13

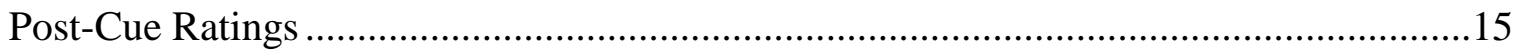

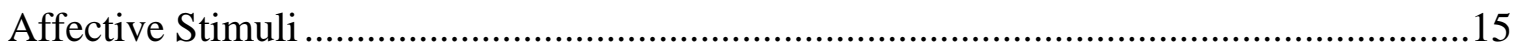

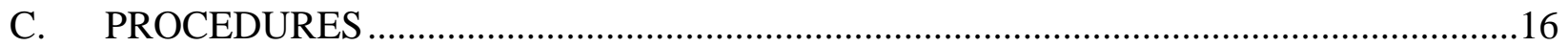

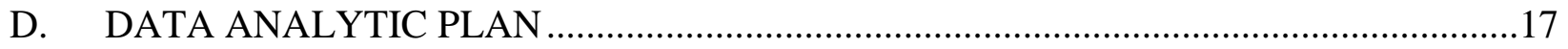

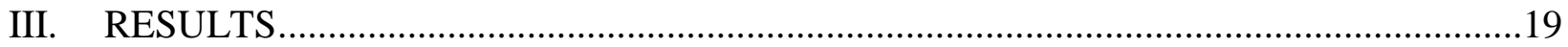

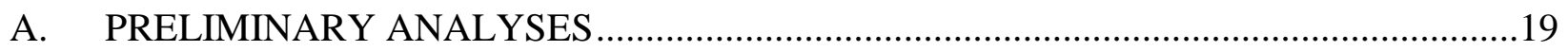

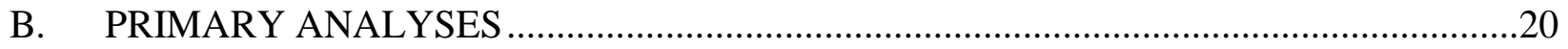

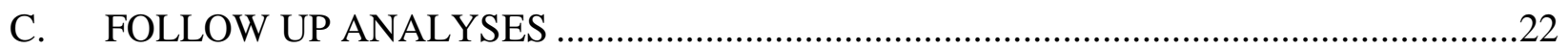

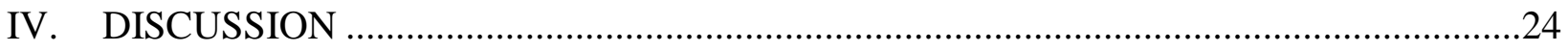

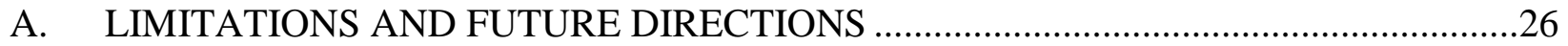

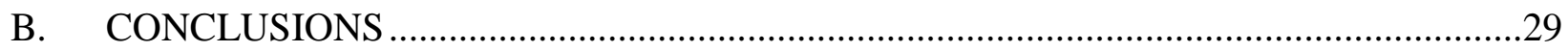

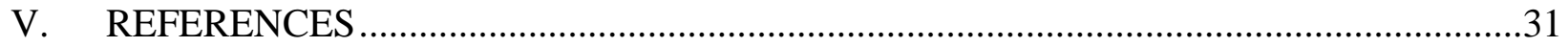


VI. APPENDIX A

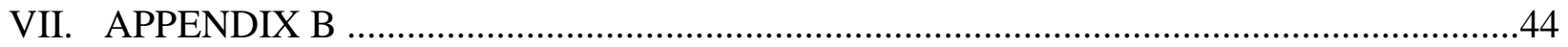

VIII. RESEARCH COMPLIANCE LETTER .................................................................46 
Psychometric Evaluation of a Standardized Set of Alcohol Cue Photographs to Assess Craving

Alcohol use remains a significant health concern with substantial individual and societal costs. The link between alcohol use and increased risks for illness and death has been well documented in the literature (Hingson, 2010). As of 2012, slightly more than half (51.8\%) of all Americans age 12 or older reported being a current drinker of alcohol, translating to an estimated 135.5 million people (Substance Abuse and Mental Health Services Administration [SAMHSA], 2013). Nearly one quarter of those same individuals also reported episodes of binge drinking (defined as 5 or more standard drinks for men and 4 or more drinks for women), which translates to approximately 59.7 million people (SAMHSA, 2013). Examining the toll of these alcohol use rates, it has been conservatively estimated that approximately 85,000 deaths attributable to alcohol consumption occur in the United States each year (Mokdad, Marks, Stroup, \& Gerberding, 2000). Moreover, several adverse medical consequences have also been directly related to alcohol consumption, including: acute and chronic pancreatitis, alcohol hepatitis, liver cirrhosis, cardiomyopathy, hypertension, arrhythmias, cerebrovascular hemorrhage, and cancers of the upper digestive tract (Chou, Grant, \& Dawson, 1998). Due to the widespread use of alcohol and its large potential to cause harm, it appears relevant and important to examine processes related to the development and maintenance of alcohol use patterns. Specifically, understanding how one's reactions to alcohol cues present in the environment might influence drinking behavior may help inform prevention and intervention strategies.

While previous research has been aimed at understanding reactions to alcohol cues, methodological limitations have unfortunately hindered what interpretations can be drawn from the results across these studies. Specifically, the ability for researchers to reliably examine reactivity to alcohol cues, particularly in the domain of craving, is limited by a lack of available 
standardized alcohol cues (Grusser, Heinz, \& Flor, 2000; Pulido, Brown, Cummins, Paulus, \& Tapert, 2010). To date, alcohol cue reactivity studies have included alcohol-related cues sourced from: (1) a mixture of standardized picture sets that were not developed with the intention of assessing alcohol cue reactivity (e.g., Normative Affective Picture System [NAPS; Stritzke, Breiner, Curtin, \& Lang, 2004], and International Affective Picture System [IAPS; Lang, Bradley, \& Cuthbert, 2008]; Drobes, Carter, \& Goldman, 2009; Pulido et al., 2010), (2) internet and stock photography sources (Carter, 2010; Drobes et al., 2009; Pulido et al., 2010), or (3) photographs created by researchers themselves (Billeux et al., 2011). Some of these studies used sets of alcohol cues in alcohol cue reactivity paradigms without any previous examination of the psychometric properties of the presented cues (Drobes et al., 2009; Carter, 2010). Other researchers provided some psychometric data related to the alcohol cues; however, aspects related to the content presented within the photographs (e.g., including non-alcohol related stimuli in the photograph; limiting the types of alcoholic beverages shown) could be improved to provide a more precise and comprehensive examination of alcohol cue reactivity (Billeux et al., 2011; Carter, 2010; Lee, Namkoong, Lee, An, \& Lee, 2006, Pulido et al., 2010).

Therefore, the current study addressed these limitations in the literature by validating a standardized set of visual alcohol cues created in a manner such that an alcoholic beverage is the primary focus in each photograph and type of beverage is balanced across cues. Specifically, the primary aim of the current study was to examine the validity and reliability of a set of alcohol cues (static images) that include images of beverages from four commonly consumed and investigated beverage types (i.e., beer, wine, liquor, and mixed drinks; e.g., Kidorf, Lang, \& Pelham,1990; Lindman \& Lang, 1986) for the purpose of inducing craving. 


\section{Alcohol Cue-Induced Craving Theory}

The concept of craving continues to be a contentious topic among today's researchers despite its long-standing history in the literature of alcohol use and alcohol cue reactivity. While there are many terms associated with the word "craving" in the literature (e.g., urge, desire, want, and need), craving is broadly viewed as a subjective and conscious experience of desire (Drummond, 2001; Kassel \& Shiffman, 1992). Specific to the purposes of the current study, craving is defined as one's "desire or urge to drink" which has been shown to be particularly associated with severity of alcohol use problems (Kramer et al., 2010). In fact, craving is thought to have a significant impact on a person's decision to approach or avoid the consumption of alcohol (Wiers et al., 2007). The current study used an integration of two theoretical models (conditioning and cognitive) as a framework for understanding the role of craving in alcohol use and alcohol use disorders.

Conditioning models of craving embrace the principles of classical and operant conditioning in learning theory. Specifically, cue-induced craving is conceptualized as a conditioned response that is produced after a cue (the conditioned stimulus) has been repeatedly paired with alcohol consumption. Importantly, within the conditioning model, craving can be elicited in the presence of a stimulus (e.g., alcoholic beverage) or situation (e.g., bar setting) that was previously associated with drinking even when alcohol consumption does not occur (Anton, 1999). In other words, ordinarily neutral stimuli, not previously associated with alcohol use, can become associated with alcohol consumption after repeated pairing and have been shown to eventually evoke alcohol-related craving by themselves (Drummond, Litten, Lowman, \& Hunt, 2000; Vollstädt-Klein, Loeber, von der Goltz, Mann, \& Kiefer, 2009). Taken together, conditioning models posit that alcohol cue-induced craving develops through classical and 
operant conditioning processes where alcohol and alcohol-related cues begin to elicit craving due to repeatedly associating the environmental cue with alcohol consumption.

Alternatively, cognitive models conceptualize craving as a subjective state mediated by the expectation that drinking will have positive effects or will improve an existing negative mood state (Anton, 1999). In this model, motivational constructs (i.e., internal and external drives that influence behavior) are widely accepted as playing an important role in associative learning, particularly when applied to substance use behaviors (Cox \& Klinger, 1988). Specifically, an individual's motivation to use alcohol (e.g., subjective craving) is intertwined with the availability of positive or negative incentives and the affective value a person places on those incentives at the time (Cox \& Klinger, 1988). By viewing craving as a motivational state influenced by cognitions, the cognitive model has allowed for a greater understanding of subjectively measured craving toward alcohol-related stimuli.

\section{Alcohol Cue-Induced Craving Methods}

Generally, both conditioning and cognitive models of craving assume a direct causal relationship between craving and subsequent drinking behavior (Drummond, Tiffany, Glautier, \& Remington, 1995); however, recent studies have called into question the simplicity of this relationship and suggest a more complex model of craving may be warranted (see Drummond et al., 2000). While this debate is beyond the scope of the present study, it is important to note that contemporary researchers often consider a combination of both conditioning and cognitive processes as being important in cue-induced craving and subsequent drinking behavior. In order to examine craving from a theoretically inclusive framework, many researchers have utilized the cue reactivity paradigm, which has garnered strong support as a means to elicit responses presumed to relate to the motivational processes involved in alcohol use (Saladin, Drobes, 
Coffey, \& Libet, 2002; Drummond et al., 1995; Reynolds \& Monti, 2013). Simply stated, the cue reactivity paradigm involves the observation and measurement of a variety of responses (described below) following exposure to a cue (Drummond et al., 2000). Within this paradigm, cues previously associated with alcohol consumption can, under certain conditions, elicit responses which may be symbolic/expressive (e.g., craving, anxiety, pleasure), physiological (e.g., drug-like, withdrawal-like, appetitive), and/or behavioral (e.g., alcohol-seeking behavior; see Drummond et al., 1995). Bridging the gap between conditioning and cognitive models, the cue-reactivity paradigm allows researchers to examine cue-elicited responses, such as craving, from multiple theoretical frameworks without assuming simple causal relationships (Drummond, 2001). Therefore, for the purpose of the current paper, the cue reactivity paradigm is viewed as a methodology that may be used to examine the relationship between alcohol cues and alcohol craving.

In previous research, methods implemented to assess cue-induced craving outcomes have varied across three main modes of assessment: physiological, behavioral, and cognitive (Drummond, 2001). Outlined by Carter and Tiffany (1999), physiological indices typically include responses controlled by the autonomic nervous system such as skin conductance, heart rate, respiration, and skin temperature; behavioral indices typically include responses related to drug use behaviors such as quantity and frequency of alcohol use as well as latency to consume alcohol; and cognitive indices typically include self-reported desire for alcohol as a means to identify and quantify an individual's subjective craving. In relation to the current investigation, assessments of an individual's subjective craving have yielded some of the most informative, adaptable, and simple methods for measuring craving (e.g., uni-dimensional scale ratings). Specifically, questions assessing subjective craving for alcohol have yielded fruitful 
interpretations despite minor variations in the phrasing of questions asked, the scale's distribution, and the descriptions of the scale's anchors (see Coffey, Saladin, Libet, Drobes, \& Dansky, 1999; Kambouropoulos \& Staiger, 2001; Payne et al., 1992; Pomerleau, Fetrig, Baker, \& Cooney, 1983; Schlauch, Geynn- Shapiro, Stasiewicz, Molnar, \& Lang, 2013; Stasiewicz et al., 1997; Rock \& Kambouropoulos, 2012 for types of methodologies implemented). Given the robust utility of assessing craving using a uni-dimensional scale, it appears to be a necessary and useful component for validating alcohol cues on the dimension of craving.

To investigate craving in this light, the cue-reactivity paradigm has shown promise for exploring the relationship between craving and alcohol-related problems (Drummond, 2000). Despite the fact that numerous studies have investigated alcohol craving using the cue-reactivity paradigm (e.g., Carter \& Tiffany, 1999; Connolly, Coffey, Baschnagel, Drobes, \& Saladin, 2009; Cooney, Litt, Morse, Bauer, \& Gaupp, 1997; Kambouropoulos \& Staiger, 2001; Lee et al., 2006; Mason, Light, Escher, \& Drobes, 2008; Monti, Rohsenow \& Hutchison, 2000; Schulze \& Jones, 2000; Rock \& Kambouropoulos, 2012), there remains a need for standardized alcohol stimuli to be available when collecting information regarding cue-induced levels of alcohol craving.

\section{Standardized Visual Alcohol Cues}

Standardized alcohol picture cues allow substance use researchers a consistent way to replicate findings, adjust methodologies, and validate measures across a broad range of study designs. Unfortunately, the ability of researchers to examine reactivity to alcohol cues has been limited by a lack of standardized alcohol cues available. To date, alcohol cue reactivity studies have been limited by several factors related to the cues selected. First, most alcohol-reactivity studies have used cues that have not been validated for the purpose of inducing alcohol craving, in large part because of a lack of research providing psychometric data regarding alcohol cue 
sets. Of the available work to date that has evaluated a set of standardized alcohol cues (Billeux et al., 2011; Lee et al., 2006; Pulido et al., 2010), there remain several limitations yet to be addressed. For example, there is the lack of standardization procedures available for assessing craving following alcohol cue sets, even with the repeated assertion that craving ratings are a necessary component for substance stimuli validation (e.g., nicotine: Carter, 2006; alcohol: Carter, 2010). Further, many previous studies have used idiographic stimuli based on each participant's preferred beverages (Coffey et al., 1999; Payne et al., 1992; Pomerleau et al., 1983; Schulze \& Jones, 2000) which is a limitation because these studies have often presented subjects with personalized beverage cues without first fully understanding the effect of personalized cues above and beyond that of standardized cues. Finally, previous studies have placed an emphasis on using alcohol cues depicting only one type of beverage (i.e. displaying only images of beer; Carter, 2010; Drobes et al., 2009), which assumes alcohol cue reactivity is not influenced by the type of beverage cue presented. Taken together, it seems apparent that despite the large number of alcohol cue-reactivity studies in the literature, there is a noted lack of empirical work focused on developing and validating a standardized alcohol cue picture set that would be useful for a variety of alcohol cue reactivity studies (Grusser et al., 2000; Lang, Bradley, \& Cuthbert, 1998; Lee et al., 2006; Stritzke et al., 2004; Wrase et al., 2002).

A review of the literature revealed three recent studies that aimed to validate a set of alcohol cues by assessing the impact of the cues on measures of "urge to drink" (Lee et al., 2006), valence and arousal (Pulido et al., 2010), or valence, arousal, and dominance (i.e., Billeux et al., 2011). In the first study, Lee et al. (2006) presented data collected from 105 Korean adults (35 alcohol dependent, 35 heavy drinkers, and 35 social drinkers; $M_{\text {age }}=38.5, S D$ $=6.9$, range $=13-23 ; 52 \%$ women) on 27 alcoholic beverage cues (i.e., soju, beer, whisky). 
Each set of cues included nine pictures of (1) a glass filled with alcoholic beverage, (2) a bottle, (3) a glass and a bottle, (4) a glass a bottle and a side dish, (5) a scene showing beverages being poured, (6) someone drinking, (7) an advertisement for each of three kinds of alcohol beverages, (8) a party scene, and (9) a signboard of a bar or alcoholic beverages displayed in a liquor store. Subjects were asked to rank-order the 6 photographs that made them most crave alcohol on a 1-6 scale. The picture that induced alcohol craving the most was ranked $1^{\text {st }}$, followed by the $2^{\text {nd }}, 3^{\text {rd }}$, $4^{\text {th }}, 5^{\text {th }}$ and $6^{\text {th }}$ ranks. Results indicated that alcohol dependent individuals reported the most alcohol craving to pictures depicting alcohol by itself and that heavy drinking individuals reported the most alcohol craving to pictures depicting drinking situations and "party scenes." No consistent characteristics of the alcohol cues chosen by social drinkers were found. While this study presents helpful information regarding the types of cues found to elicit the greatest amount of craving among different categories of drinkers, the interpretation of the results were limited by the inclusion of regionally specific drinking cues, the omission of valence and arousal ratings following cue presentation, the method in which craving was operationalized (i.e., rank ordering rather than absolute levels of craving), and incomplete evaluation of validity and reliability.

In the second study, Pulido et al. (2010) provided validation data collected from 82 "nondrinking" (i.e., 10 or fewer lifetime drinking experiences) adolescents and emerging adults in the United States $\left(M_{\text {age }}=18.1, S D=2.2\right.$, range $=13-23 ; 52 \%$ women; $41 \%$ White $)$ on 26 alcoholic beverage cues (i.e., beer, wine, and hard liquor) and 26 non-alcoholic cues (e.g., soft drinks, juice, water, and milk). Beverage stimuli were drawn from larger set of 120 photographs (60 alcoholic and 60 non-alcoholic) which were obtained primarily from advertisements, "amateur" photographs, NAPS, IAPS, and the internet. Of note, some photographs depicted people 
consuming the beverage, which has been reported to be a limitation of subsequent alcohol cue investigations due to the introduction of additional variance (Drobes et al., 2009). Further, the inclusion of advertisements with brand name information highlighted may also affect reactions. Results indicated that subjects rated non-alcohol pictures as more pleasant on the dimension of valence than they rated alcohol-related pictures. Results also indicated that arousal ratings were not significantly different following the two picture types. While this study presents some helpful data regarding a potential set of standardized alcohol cues, the interpretation of the results of this validation study were limited by the inclusion of alcohol cues in which the prime focus is not on an alcoholic beverage, the omission of craving ratings following cue presentation, and the use of a sample which consisted of youth and college students primarily below the legal alcohol drinking age with little or no drinking experience without an alcohol-using comparison group.

In the third study, Billeux et al. (2011) presented data regarding alcohol-related cues from 101 Swiss and French adults $\left(M_{\mathrm{age}}=27.30, S D=8.59\right.$, range $=19-60 ; 67 \%$ women $)$ who reported various levels of drinking patterns. The picture set consisted of 60 alcohol photographs, drawn from a larger set of 200 , and included photographs displaying various types of alcoholic drinks (e.g., beer, wine, hard liquor, cocktails), scenes depicting drinking behaviors (e.g., people drinking, someone making a cocktail, people eating a meal with drinks present), and photographs without alcohol present (e.g., a corkscrew). The selection of photographs is contrary to the typical alcohol cue-reactivity protocol in which the aim is to only include pictures with a direct portrayal of alcohol or alcohol consumption so as to reduce variance attributed to factors other than alcohol, and thus may not be appropriate for many types of studies. The researchers found that risky drinkers reported higher levels of arousal and dominance, but not valence, following 
presentation of alcohol cues than non-risky drinkers. The results of this evaluation were limited by the content of the cues, the lack of control/neutral stimuli, the lack of craving assessment, and the lack of alcohol risk group differences in valence ratings following the alcohol cues.

Taking together the review of the studies by Lee et al. (2006), Pulido et al. (2010) and Billieux et al. (2011) with research findings supporting the relationship between cue-elicited craving, there remains room for improvement related to inclusion and exclusion criteria of alcohol stimuli in alcohol cue studies as well as the procedures for standardization of alcohol cues (Curtin, Barnett, Colby, Rohsenow, \& Monti, 2005). Specifically, it appears reasonable that assessing subjective alcohol craving in response to alcohol cues may be an important component in determining the utility of a set of alcohol cues for use in cue reactivity studies (Carter, 2010). Additionally, investigations into whether the type of alcohol cue is important in understanding cue-induced alcohol craving particularly within a drinking population appear warranted. Furthermore, despite research showing that personally relevant stimuli may elicit stronger physiological and emotional effects than standardized materials (Cinciripini et al., 2006; Payne et al., 1992) and that personalized cues may augment physiological responding in cue reactivity paradigms (Cook, Melamed, Cuthbert, McNeil, \& Lang, 1988), the examination of these differences using a set of alcohol cues, standardized on the domain of craving, appears to be a critical next step toward understanding the potential impact of personalized cues on subjective craving.

\section{Current Study}

The current study aimed to evaluate the psychometric properties for a set of alcohol cues for use in alcohol cue reactivity studies involving non-alcohol dependent samples. The present study expanded upon previous alcohol cue standardization studies (Billeux et al., 2011; Lee et 
al., 2006; Pulido et al., 2010) by (1) examining a set of alcohol beverage cues that adequately excluded extraneous influences (e.g., advertising effects, branding effects, social depictions, gender stereotypes, and assumed associations to alcohol use); (2) providing a more comprehensive assessment of validity and reliability; (3) adding the measurement of subjective craving alongside valence and arousal following presentation of each cue; and (4) examining a set of alcohol cues that include the most commonly consumed beverages (e.g., beer, wine, liquor, mixed drinks). Future research will benefit from the development and examination of this standardized set of alcohol beverage cues.

Psychometric evaluation of alcohol cues. The present study examined factor structure, internal consistency, and convergent and discriminant validity as ways to evaluate the psychometric properties of the alcohol cues. First, the factor structure of the craving ratings following alcohol cues were examined using exploratory factor analysis. Given that this was the first examination of these photos and there was no previous research from which to draw hypotheses, no hypotheses were specified regarding whether there would be a unitary factor structure or more than one factor (e.g., by beverage type). The results of the exploratory factor analyses were used to determine the computation of mean craving rating scores for the remaining analyses. In addition, internal consistency of the craving ratings following alcohol cues was evaluated based on the resulting factor structure.

To test convergent validity, we examined the associations between alcohol cue-elicited craving ratings and related constructs. It was hypothesized that craving ratings following alcohol cues would be positively correlated with general craving for alcohol, self-reported levels of pastyear hazardous alcohol use, and three facets of behavioral activation. To test incremental validity, it was hypothesized that craving ratings following alcohol cues would continue to be 
positively correlated with general craving for alcohol when controlling for behavioral activation and self-reported levels of hazardous alcohol use. Also, it was hypothesized that craving ratings following alcohol cues would be positively correlated with self-reported levels of hazardous alcohol use when controlling for behavioral activation and general craving for alcohol. As tests of discriminant validity, it was hypothesized that valence, arousal, and craving ratings would be higher following alcohol cues compared to neutral cues. Further, it was hypothesized that craving for alcohol following the alcohol cues should be weakly correlated or unrelated to behavioral inhibition or severity of nicotine dependence.

\section{Method}

\section{Participants}

The sample consisted of 195 volunteers drawn from a larger sample of 350 volunteers recruited through Mechanical Turk $^{\circledR}\left(\right.$ MTurk $\left.^{\circledR}\right)($ See "Data Cleaning” for information about procedures for determining valid participants). Participants over the age of 18 , living in the United States, and demonstrating a “quality rating” of .90 or better in MTurk ${ }^{\circledR}$ (reflecting accurate and complete responding in most instances) were recruited to participate in the study and interested participants were transferred to the online consent form on Qualtrics ${ }^{\circledR}$.

Participants were excluded if they reported alcohol consumption within four hours of the study to reduce likelihood of recent alcohol ingestion affecting responses. Additionally, participants who self-identified as alcoholic, who were currently recovering or abstaining from alcohol use, or who had not consumed alcohol in the past 30 days were asked to exclude themselves from participation. Participants were predominately male (74.4\%) with a mean age of 32.19 years ( $S D=10.63$; range from 20 to 69 years). The majority of participants identified as Asian, Asian-American, or Pacific Islander (56.4\%) or White, Caucasian, or Non-Hispanic 
(34.9\%). Other ethnicities included those who identified themselves as Black Hispanic/Latino (1\%), White Hispanic/Latino (1\%), African-American/Black, Non-Hispanic (0.5\%), and Other (4.6\%). Education and occupation data indicated that a majority of the participants had a twoyear college degree or higher $(77.4 \%)$ and were currently employed $(82.1 \%)$. Regional information showed that the majority of participants identified as residing in the South (43.1\%) compared to the Midwest (28.7\%), the West (13.3\%), or the Northwest (12.3\%). See Table 1 for a complete summary of participant demographic information.

\section{Measures and Stimuli}

\section{Self-report questionnaires.}

Demographics. Participants completed a demographic information form to help describe the sample characteristics and identify potential covariates. Individuals were asked to answer questions assessing gender, age, race/ethnicity, region of residence, marital status, employment, education, current student status, living arrangements, income, sexual orientation, and handedness. Drink type preference was assessed by asking participants to rate their preference for drinking common alcoholic beverages (beer, wine, hard liquor, mixed drinks, wine cooler, fortified wine, liqueurs, champagne) on a scale from 1 to 8 using each number only once. In addition, participants were asked about how long it had been since consuming their most recent alcoholic beverage.

Desires for alcohol questionnaire (DAQ). The DAQ contains 14 items that assess intentions to drink alcohol, desires to consume alcohol, anticipation of positive outcomes from drinking, and anticipation of relief of negative affect or alcohol withdrawal. Items were rated on a 7-point Likert-type scale from 1 (not at all likely) to 7 (extremely likely). Overall, the DAQ has demonstrated concurrent validity with measures of hazardous drinking (i.e., the 
AUDIT) and good reliability among individuals with and without an alcohol use disorder (Kramer et al., 2010). The DAQ has also shown high internal consistency, with a Cronbach's alpha between .88 and .96 (Kramer et al., 2008; Love, James, Willner, 1998). In the present sample, the mean DAQ total score was $50.27(S D=15.23)$ and Cronbach's alpha was .89.

Alcohol use disorder identification test (AUDIT). The 10-item AUDIT (Babor, Higgens-Biddle, Saunders, \& Monterio, 2001) was used to assess quantity and frequency of alcohol use, dependence symptoms, and negative consequences of alcohol use over the past year. The AUDIT can be used to identify hazardous drinkers as well as drinkers with alcohol use disorders. Both the test-retest reliability $(r=.86)$ and internal consistency $(\alpha=.83)$ have been shown to be high (Hays, Merz, \& Nicolas, 1995). The mean score in the present sample was $12.79(S D=8.17)$ and Cronbach's alpha was .87 .

\section{Behavioral inhibition system / behavioral activation system (BIS/BAS). The}

BIS/BAS (Carver \& White, 1994) was used to assess individual differences in the sensitivity and regulation of both aversive motives (behavioral avoidance/inhibition system; BIS) as well as appetitive motives (behavioral approach system; BAS) on a 1 (very true of me) to 4 (very false of me) scale. The BIS, which assesses punishment sensitivity or anticipation of punishment, is comprised of a single scale which has demonstrated sufficient internal consistency $(\alpha=.74$, Carver \& White, 1994). The BAS scale is comprised of three subscales: drive (the persistent pursuit of desired goals; BASD); fun seeking (both a desire for new rewards and a willingness to approach a potentially rewarding event on the spur of the moment; BASF); and reward (positive responses to the occurrence or anticipation of reward; BASR) and each were shown to also have sufficient internal consistency, $\alpha=.76, .66$, and .73, respectively (Carver \& White, 1994). In the present sample, the mean BIS score was $19.28(S D=3.54)$ and Cronbach's alpha was .69. The 
mean BASD score was $11.50(S D=2.51)$ and Chronbach's alpha was .78. The mean BASF score was $11.27(S D=2.43)$ and Chronbach's alpha was .72. The mean BASR score was 16.08 $(S D=3.05)$ and Chronbach's alpha was .82.

Fagerström test of nicotine dependence (FTND). The FTND (Heatherton, Kozlowski, Frecker, \& Fagerstrom, 1991) is a 6-item self-report measure of nicotine dependence. The FTND can be used to predict continued smoking and has been shown to be positively correlated with years of smoking and biochemical indices of smoking. The FTND has demonstrated fair internal consistency ( $\alpha=.61$; Heatherton et al., 1991). In the present sample, the mean FTND score was $4.27(S D=2.00)$ and Cronbach's alpha was .49 .

Post-cue ratings. The Self-Assessment Manikin (SAM) is a pictographic analogue scale designed for nonverbal assessment of pleasure, arousal, and dominance (Bradley \& Lang, 1994). Typical of cue reactivity research, participants were asked to make ratings of valence and arousal following all presented images on a 9-point scale with a rating of 1 indicating a low value (low pleasure, low arousal) and 9 indicating a high value (high pleasure, high arousal) on each dimension. This subjective rating of valence and arousal is used nearly universally in standardized cue reactivity research and has been shown to have valid and reliable psychometric properties in measuring affective reactions (e.g., Billeux et al., 2011; Carter, 2010; Grusser et al., 2000; Pulido et al., 2010; Wrase et al., 2002). Additionally, participants were asked to make craving ratings following all presented images on a 9-point scale $(1=$ no craving or desire for alcohol; 9 = intense craving or desire for alcohol) as this format allows researchers a method for examining craving using a uni-dimensional scale.

\section{Affective stimuli.}


Alcohol stimuli. To assess responses to alcohol cues, fifteen photographs from each of four alcohol beverage categories (i.e., beer, wine, hard liquor, and mixed drinks) were created using a Samsung L200 digital camera at 10.2 megapixel (3648 x 2736) resolution. Photographs were reduced in resolution to $800 \times 600$ or its transposed equivalent to ensure that picture size remained consistent with the photographs included in the IAPS database (Lang et al., 2008). All cues were created within a simulated bar setting (i.e., bar lab) with a neutral, simple background and with primary focus on the alcoholic beverage. Each alcohol cue was photographed in three positions: (1) while being poured from a bottle into a glass, (2) while resting on the bar counter in a glass, and (3) while resting on a countertop behind a bar in a glass (see Appendix B for example photographs). For each type of alcohol, there were five variations of the beverage presented, each presented three times in the three positions.

IAPS stimuli. To assess responses to already standardized affective cues, 12 pictures were selected from each of the three IAPS categories (i.e., positive, negative, neutral) based on their normative properties (Lang et al., 2008). The positively-valenced cues (e.g., couples embracing, wealth, adventure) used were composed of IAPS numbers 4652, 4658, 4659, 4660, 4670, 4676, 4695, 7330, 8502, 8510, 8030, and 8200. The negatively-valenced cues (e.g., snakes, bears, threat) included IAPS numbers 1050, 1120, 1300, 1321, 1525, 1931, 6230, 6260, 6244, 6250, 6300, and 6510. The neutral cues (e.g., hairdryer, lamp, and clock) included IAPS numbers 7000, 7010, 7020, 7030, 7041, 7050, 7052, 7055, 7175, 7190, 7217, and 7235.

\section{Procedures}

As previously mentioned, participants were recruited through MTurk $^{\circledR}$. MTurk ${ }^{\circledR}$ is a website sponsored by Amazon.com that brings together "people and tools" to "enable task creation, labor recruitment, compensation, and data collection" (Burmester, Kwang, \& Gosling, 
2011). According to Burmester et al. (2011), data collected using MTurk ${ }^{\circledR}$ is similar in reliability to data collected using traditional data collection methods, and results in a diverse sample compared to typical samples utilized in research. People register as "requesters" (in our case, researchers) or "workers" (in our case, paid participants). From this site, requesters create and post surveys. Workers can browse available surveys and are paid upon successful completion of a selected survey.

Eligible and interested participants were redirected to a Qualtrics ${ }^{\odot}$ link and presented with an informed consent form. After indicating consent, participants were redirected to the Qualtrics $^{\circledR}$ online survey. Participants were then asked to complete measures online examining demographics, desire for alcohol, hazardous alcohol use severity, behavioral inhibition and activation, and smoking behaviors. After all self-report questionnaires were administered, 15 pictures from each of four alcohol beverage categories (i.e., beer, wine, hard liquor, and mixed drinks) and 12 pictures from each of the IAPS categories (e.g., positive, negative, neutral) were presented in randomized order. The picture series contained a total of 96 pictures. Participants were asked to provide valence, arousal, and craving ratings following each presented image. Based on piloting data, it was estimated that participants would take no more than 25 minutes to complete the study (i.e., 14.5 responses per minute). Based on the average current rate for participation provided by MTurk ${ }^{\circledR}$, participants were compensated $\$ 0.69$. It should be noted that the average completion time for the present study was longer than anticipated, at 39.27 minutes $(S D=17.80$ minutes $)$

\section{Data Analytic Plan}

Prior to the primary data analyses, preliminary analyses were conducted to complete data cleaning and to check for violations of statistical assumptions. Specifically, frequencies and descriptive analyses were examined to determine data errors and appropriate coding of variables 
as well as to remove cases with random responding, ineligible cases, and outliers $(3+$ standard deviations outside the mean). Additionally, for the principle component analysis, the assumptions of normality, linearity, homoscedasticity, sampling adequacy, and sphericity were investigated. Among the paired t-tests, the assumptions of normality were investigated. Finally, among the Pearson correlations, normality, linearity, and homoscedasticity were investigated.

Next, data primary analyses were conducted. First, a principal component analysis (PCA) was used to test the factor structure of the alcohol craving ratings from the 60 alcohol cues (15 beer, 15 wine, 15 hard liquor, 15 mixed drinks). In addition, internal consistency of the craving ratings following alcohol cues was evaluated for each resulting factor using Chronbach's alpha. To assess convergent validity of the alcohol cue picture set, a series of Pearson correlations were conducted between craving ratings following the alcohol cues and general craving for alcohol as measured by the DAQ, self-reported hazardous alcohol use as measured by the AUDIT, and behavioral activation as measured by the BASR, BASF, and BASD. To assess incremental validity, partial correlations were conducted between craving ratings following alcohol cues and general craving for alcohol, controlling for self-reported hazardous alcohol use and behavioral activation subscales. Also, a partial correlation was conducted between craving ratings following alcohol cues and self-reported levels of hazardous alcohol use when controlling for behavioral activation and general craving for alcohol. Next, as a manipulation check of the cue reactivity model prior to discriminant validity analyses, paired samples $t$-tests were conducted to examine mean differences across valence and arousal ratings following positive, negative, and neutral pictures. To assess discriminant validity of the craving ratings following the alcohol cues, paired-sample $t$-tests were conducted to examine the effect of picture type (alcohol vs. neutral) on valence, arousal, and craving ratings following cue 
exposure. Additionally, correlations were conducted between craving ratings following the alcohol cues and behavioral inhibition as measured by the BIS and severity of nicotine dependence as measured by the FTND. Finally, differences in mean craving ratings in age, gender, and race/ethnicity were tested and descriptive data were provided.

\section{Results}

\section{Preliminary Analyses}

Data cleaning. A single error occurred in the data in which 10 participants provided two ratings of arousal following a negative cue (an impossible feature for the rating scale). Individuals who provided two ratings on their arousal scale for the image were removed from subsequent analyses. The question assessing hours since participants consumed alcohol was originally coded using string variables in which participants could input text. In order to convert the string variables into a true scale, the words "hour," "hours," "hr," "hrs," and "hr ago" were removed. Reported "days" and "weeks" were converted into their respective times in hours. To aid in removing potentially random responders, all analyses were conducting using only cases in which all picture ratings were completed and the participant reached the study's debriefing page. Of the total sample size $(N=350)$, only 291 provided ratings on all pictures and only 218 completed the study by reaching the debriefing form at the end of the questionnaire packet. Individuals who reported consuming alcohol less than four hours prior to the start of the experiment were considered invalid and were removed. A total of 15 participants reported consuming alcohol within the past four hours ( 0 hours, $n=4 ; 1$ hour, $n=4 ; 2$ hours, $n=5 ; 3$ hours, $n=2$ ) and were subsequently removed, leaving a total of 203 participants. Participants that fell outside three standard deviations of mean completion time for the study were removed. There were no extreme outliers on age, FTND, AUDIT, BIS, BAS, DAQ, cue categories (i.e., positive, negative, neutral, alcohol), or ratings of arousal, valence, or craving. There were, 
however, eight outlying data points for mean completion time for the study, leaving a total of 195 participants in the final dataset. See Table 1 for demographic and questionnaire frequencies.

Checking statistical assumptions. For the PCA, the assumptions of normality linearity, homoscedasticity, sampling adequacy (.98) exceeding the recommended value of .60 (Kaiser,1970), and sphericity $(p<.0001)$ were satisfied. The assumptions of normality (skew = .38 to .30 ; kurtosis $=-.85$ to .68$)$ and linearity, $(p<.05)$ for the paired $t$-test comparisons were satisfied. Across the Pearson correlations, the assumption of normal distribution (skew $=-.33$ to .54 ; kurtosis $=-1.75$ to .66$)$, linearity $(p<.05)$, and homoscedasticity were met.

\section{Primary Analyses}

Factor structure. An obliquely-rotated PCA factor analysis including the craving ratings following the 60 alcohol cues revealed the presence of two components with Eigenvalues exceeding 1.0. The first factor (Eigenvalue $=49.67$ ) explained $82.79 \%$ of the variance, while the second factor $($ Eigenvalue $=1.19$ ) accounted for only $1.99 \%$ of the variance. However, evidence suggested that only the first factor should be retained. First, inspection of the scree plot revealed a clear break after the first component. Further, parallel analysis, a procedure that statistically determines the break in a scree plot (Horn, 1965; Longman, Cota, Holden, \& Fekken, 1989), suggested that a one component solution best fit the data. As shown in the pattern matrix in Table 2, all ratings loaded positively on this factor and above the a priori cutoff of .40 (Spector, 1992).

Internal consistency. The internal consistency for the retained factor was excellent, with a Cronbach's alpha of .99.

Convergent validity. Craving for alcohol following alcohol cues was positively correlated with general craving for alcohol, $r(195)=.75, p<.001$; self-reported levels of hazardous alcohol use, $r(195)=.61, p<.001$; and drive and fun-seeking components of 
behavioral activation, $r(195)=.24, p<.001$ and $r(195)=.25, p<.001$, respectively. Craving ratings following alcohol cues were not significantly correlated with the reward component of behavioral activation, $r(195)=-.015, p=.84$.

Incremental validity. Controlling for self-reported hazardous alcohol use and behavioral activation, craving for alcohol following alcohol cues was still positively correlated with general craving for alcohol, $r_{\mathrm{p}}(189)=.58, p<.001$. Controlling for general craving for alcohol and behavioral activation, craving for alcohol following alcohol cues was still positively correlated with self-reported levels of hazardous alcohol use, $r_{\mathrm{p}}(189)=.26, p<.001$.

Discriminant validity. The cue-reactivity manipulation check supported the use of neutral cues as comparisons for alcohol cues such that valence and arousal ratings following positive photos were significantly higher than neutral photos, $t(194)=18.46, p<.001$ and $t(194)$ $=22.10, p<.001$, respectively. Valence and arousal ratings following negative photos were significantly lower than neutral photos, $t(194)=13.74, p<.001$ and $t(209)=4.32, p<.001$, respectively. Additionally, the valence, arousal, and craving ratings following alcohol cues were all highest compared to neutral cues, $t(194)=12.86, p<.001, t(194)=14.31, p<.001$, and $t(194)=16.26, p<.001$, respectively (see Table 4 for subjective cue ratings).

Lastly, as hypothesized, craving following alcohol cue photos was not significantly correlated with behavioral inhibition, $r(195)=-.084, p=.24$, or severity of nicotine dependence, $r(195)=.10, p=.92$.

Demographics and craving ratings. Craving was significantly negatively correlated with age, $r(195)=-.44 p<.001$. Craving for alcohol following the alcohol cue photos was significantly higher among men $(M=5.47, S D=2.57)$ compared to women $(M=4.54, S D=$ 2.63), $F(1,193)=4.77, p=.030$, partial $\eta^{2}=.024$. The relationship between gender and craving 
ratings following alcohol cues was found to be non-significant when controlling for AUDIT scores, $F(1,192)=.097, p=.76$, partial $\eta^{2}=.001$.

The relationship between craving for alcohol following alcohol cues and ethnicity was found to be significant, $F(2,192)=76.06, p<.001$, partial $\eta^{2}=.44$. Pairwise comparisons showed that craving for alcohol following alcohol cues was significantly lower among participants who identified as "White (Caucasian/non-Hispanic)", $M=2.91, S D=2.06$, compared to "Asian, Asian American, or Pacific Islander", $M=6.64, S D=1.72, p<.001$, or "Other" (all remaining ethnic categories collapsed due to small cell sizes); $M=5.37, S D=2.87$, $p<.001$. Craving for alcohol following alcohol cues was also significantly lower among participants who identified as "Other" compared to "Asian, Asian American, or Pacific Islander", $p=.014$.

The relationship between craving for alcohol following alcohol cues and ethnicity when controlling for hazardous alcohol use and general craving was also found to be significant, $F(4$, $190)=26.25, p<.001$, partial $\eta^{2}=.21$. Pairwise differences showed that craving for alcohol following alcohol cues was significantly lower among participants who identified as "White (Caucasian/non-Hispanic)", $M=4.02, S E=.20$, compared to "Asian, Asian American, or Pacific Islander", $M=5.97, S E=.151, p<.001$, or "Other" (all remaining ethnic categories collapsed due to small cell sizes); $M=5.30, S E=.36, p=.002$. Differences in craving for alcohol following alcohol cues among participants who identified as "Other" compared to "Asian, Asian American, or Pacific Islander" were not significant, $p=.86$.

\section{Follow-up analyses}

Given that the types of beverages included in cue reactivity paradigms have varied across standardization attempts (e.g., Billieux et al., 2011; Lee et al., 2006; Pulido et al., 2010), follow- 
up analyses were conducted to examine whether craving for alcohol following alcohol cues varied by beverage category (i.e., beer, wine, hard liquor, mixed drinks) using a repeated measures ANOVA. The results for the ANOVA indicated a significant effect of beverage, Wilks's $\Lambda=.91, F(3,192)=6.54, p<.001, \eta^{2}=.09$. Post hoc comparisons using the Bonferroni correction revealed that craving for alcohol following beer cues was significantly higher than for wine, $p=.005$, hard liquor, $p<.001$, and mixed drink cues, $p=.016$. There were no significant differences in craving for alcohol following cues for wine versus mixed drinks, $p>.99$, wine versus hard liquor, $p>.99$, or mixed drinks versus hard liquor, $p=.13$. Further, the craving ratings following alcohol cues were found to be internally consistent for each drink type category (beer, wine, hard liquor, and cocktails; see Table 5).

Additionally, given that alcohol preference has been reported to be an important component of alcohol cue-elicited craving (e.g., Coffey et al., 1999; Payne et al., 1992; Pomerleau et al., 1983; Schulze \& Jones, 2000), a follow-up one-way ANOVA sought to examine the impact of drink type preference on craving for alcohol following alcohol cues. Restricting the sample to only those that preferred beer, wine, hard liquor, or mixed drinks, no significant differences were found between drink type preference and craving for alcohol following alcohol cues, $F(3,130)=.75, p=.53, \eta^{2}=.017$. Additional one-way ANOVAs were conducted to examine differences in craving ratings for alcohol following specific beverage type cues by drink type preference. It was found that drink type preference did not significantly predict differences in craving ratings for alcohol following beer cues, $F(3,130)=1.67, p=.18, \eta^{2}$ $=.037$, wine cues, $F(3,130)=.401, p=.75, \eta^{2}=.01$, mixed drink cues, $F(3,130)=.60, p=.62$, $\eta^{2}=.014$, or hard liquor cues $F(3,130)=.64, p=.59, \eta^{2}=.015$. 


\section{Discussion}

Across the alcohol-cue reactivity literature, methodological limitations have hindered what interpretations can be drawn from the results across studies. As such, it is difficult to understand what the literature to date tells us about people's reactions to alcohol cues, despite the wide use of these cues in cue reactivity paradigms. The current study aimed to address this limitation by examining the psychometric properties of a standardized set of visual alcohol cues to assess alcohol craving. Specifically, the present study expanded upon previous alcohol cue standardization studies (Billeux et al., 2011; Carter et al., 2010; Pulido et al., 2008) in four ways: (1) reducing the likelihood of extraneous factors influencing reactivity by excluding aspects such as advertising logos, people, and scenes in which alcohol was not the sole focus of the cues; (2) including related measures to allow for a more comprehensive assessment of validity and reliability related to alcohol craving; (3) assessing subjective craving alongside measures of valence and arousal following each cue; and (4) by including the four most commonly consumed beverages (beer, wine, liquor, and mixed drinks) in the alcohol cue set. Overall, results supported the validity and reliability of the alcohol cue set among non-dependent alcohol users.

Results from the current study provide evidence that reported level of craving following this set of alcohol cues is a supported method for measuring the dimension of craving among non-dependent alcohol users in a cue reactivity paradigm. Specifically, using principal component analysis, the craving ratings reported following the alcohol cues demonstrated good structural properties, with the one-factor model providing the best fit to the data. This study's findings also expand upon previous investigations examining the psychometric properties of alcohol cues (e.g., Billeux et al., 2011; Carter, 2010; Pulido et al., 2008) by examining convergent validity between craving for alcohol following alcohol cues and general craving for alcohol, hazardous alcohol use, and behavioral activation sensitivity, as well as discriminant 
validity between craving for alcohol following alcohol cues and behavioral inhibition, severity of nicotine dependence, and alcohol craving following neutral cues. Additionally, despite finding that the structural properties were best explained by a single factor, follow-up analyses investigating the effect of drink type cues on craving for alcohol revealed that beer cues were associated with significantly higher alcohol craving ratings than craving ratings following cues of wine, hard liquor, and mixed drinks. Restricting the sample to only those that preferred beer, wine, hard liquor, or mixed drinks, follow-up analyses revealed that drink type preference did not significantly predict these differences in craving ratings for alcohol following beer, wine, mixed drinks, or hard liquor cues.

Examination of the validity indices of the cue-elicited alcohol craving ratings revealed that the overall alcohol cue set demonstrated excellent internal consistency and strong convergent and discriminant validity. Supporting the convergent validity, large effects were observed between craving ratings following alcohol cues and theoretically relevant measures such as general craving for alcohol (assessed using the DAQ) and self-reported past year hazardous alcohol use (assessed using the AUDIT). Small effects were also found between craving ratings following alcohol cues and fun-seeking behavior (desire for new rewards and willingness to impulsively seek out rewarding outcomes) and behavioral drive (the persistent pursuit of desired goals), assessed using the BIS/BAS. This pattern of responding was expected as general craving for alcohol and hazardous alcohol use have been shown to have large effects on alcohol craving following alcohol cues (Carter, 2010; White \& Staiger, 1991) whereas behavioral drive has been shown to have a weak but significant effect on craving for alcohol following alcohol following exposure to alcohol cues (Franken, 2002) and fun-seeking has been 
shown be conceptually related by its significant yet weak effect on associated levels of harmful alcohol use (Franken \& Muris, 2006; Yen, Ko, Yen, Chen, \& Chen, 2009).

Support for the discriminant validity of the alcohol cue set was also observed such that measures that theoretically should not correlate with the post-alcohol cue craving ratings were either weakly related or unrelated; for example, behavioral inhibition (punishment sensitivity; assessed using the BIS/BAS) and severity of nicotine dependence (assessed using the FTND) did not correlate with craving or "urge to drink" following alcohol cues. Providing additional support for the discriminant validity of the alcohol cue set in inducing self-reported craving, alcohol craving ratings assessed following the alcohol cues were significantly higher than alcohol craving ratings assessed following neutral cues. These findings were also as expected as valence, arousal, and craving ratings following alcohol cues have previously been found to be higher than neutral cues (Drobes, et al., 2009; Carter, 2010). Additionally, given that previous research has only shown substantial overlap between alcohol and smoking cues' ability to elicit craving in addicted populations (Drobes, 2002), and given that the present sample was not recruited based on alcohol dependence or nicotine dependence, severity of nicotine dependence was not expected to be related to alcohol craving following alcohol cues, which is consistent with our findings. Taken as a whole, these findings provide support that the set of alcohol cues created in the current study are valid and reliable for use in alcohol cue reactivity paradigms and may even provide researchers with a breadth of application possibilities.

\section{Limitations and Future Directions}

Of course, there were some limitations in the methods and administration of the alcohol cue set developed in the present study. First, it is possible that the alcohol craving ratings assessed following the alcohol cues were limited in their ability to capture the multidimensional 
model of alcohol craving proposed to be ideal in cue reactivity studies (Connolly et al., 2009; Schulze \& Jones, 2000). Due to the use of a uni-dimensional model of craving (i.e., desire or urge to use alcohol) used in this study, the impact of avoidance motivated behaviors (e.g., desire or urge to avoid the use of alcohol) remains unknown. Also, given that the present study assessed alcohol craving following alcohol cue presentation, the study findings may conceptually differ from studies that have participants rate their craving for the depicted alcoholic beverage (e.g., Lee et al., 2006). Future research should aim to examine and clarify the role of craving dimensions within cue-reactivity paradigms as well as aim to standardize the manner in which alcohol craving is assessed within alcohol cue-reactivity studies.

Furthermore, it should be noted that the generalizability and interpretability of the current study were limited in three ways. First, the restricted sample size in the current study's PCA ( $N$ =195) was slightly below recommended levels for the number of participants per variable for sufficient power $(N=215$; Horn, 1965; Longman et al., 1989). However, the strong factor loadings found in the study does not support the notion that the study was underpowered for exploratory factor analyses. Second, the ethnic majority of the sample, "Asian, Asian American, or Pacific Islander,” reported significantly higher levels of alcohol craving following alcohol cues than other ethnicities (i.e., "White (Caucasian/non-Hispanic)" and "Other") and significantly higher levels of alcohol craving than participants who identified as "White (Caucasian/non-Hispanic)" when controlling for hazardous alcohol use and general craving for alcohol. Due to these findings, it remains unclear what the implications are for the psychometrics properties of measuring alcohol craving following alcohol beverage cues across different ethnic groups. Third, cell sizes across drink type preference categories were small (range $=6-73$ ), making it difficult to draw conclusions regarding the impact that drink type 
preference may have had on the elicitation of craving following presentation of an individual's preferred alcoholic beverage. Future studies would benefit from the inclusion of a larger sample of participants to provide necessary power for factor analyses involving all 60 images contained in the current set of cues. Moreover, future analyses may benefit from reduction of the alcohol cue photograph set presented here so as to reduce the potential for redundancy and fatigue effects. Additionally, future studies would benefit from a more equal distribution of ethnicity so as to better explain the potential role that ethnicity may play in determining an individual's rating of craving following alcohol cues and to increase the generalizability of findings. Similarly, given that most drink type preference groups were small, including equal groups of participants based on drink type preference in future studies may allow for a greater understanding of how an individual's alcohol preference may affect reactivity to alcohol cues.

Lastly, the presented alcohol cues may not be suitable for all cue-reactivity studies (e.g., neuroimaging studies). As highlighted by the methodology of Pulido et al. (2010), stimuli used in neuroimaging studies typically need experimental as well as neutral stimuli carefully matched on factors such as valence, arousal, image complexity, brightness, and hue. Future studies would benefit from creation and assessment of neutral cues in this fashion as they could complement the alcohol cues presented here and enable use of the alcohol cues in a wider range of study paradigms. Also, it should be noted that since the study did not examine additional forms of validity and reliability (e.g., test-retest reliability, predictive validity) that should be explored in future research. Moreover, given that the current study did not directly recruit individuals with alcohol use disorders, the generalizability of the findings may be limited for use within alcohol dependent samples. Future studies would benefit from expanding the study design to include 
test-retest of alcohol craving following the alcohol cues as well as expanding the sample to include individuals with alcohol use disorders.

\section{Conclusions}

This study is the only known alcohol cue standardization study to date to validate a set of visual alcohol cues created in a manner such that an alcoholic beverage is the primary and sole focus in each alcohol cue. It also appears to be the first standardization attempt where cues are balanced to include images of beverages from four commonly consumed and investigated beverage types (i.e., beer, wine, liquor, and mixed drinks; e.g., Kidorf, Lang, \& Pelham,1990; Lindman \& Lang, 1986) for the purpose of inducing alcohol craving. Expanding upon earlier work (e.g., Billeux et al., 2011; Lee et al., 2006; \& Pulido et al.; 2010), the presented set of alcohol cues showed strong validity and reliability in the assessment of alcohol craving following alcohol cues. This study further built upon previous investigations in the assessment of convergent and discriminant validity that previous studies either omitted or neglected to assess. The examination of how craving ratings following alcohol cues relate to general craving and self-reported hazardous alcohol use allows researchers a greater insight into the effect that the cue standardization procedures may have had within this study.

Given that no previous study has examined the factor structure, reliability, and validity of a set of alcohol cues using the standardization format of this paper, the implications for such findings appear to be potentially far-reaching given the widespread use of photographic alcohol stimuli in research studies. Using the presented alcohol cues in future cue-reactivity or related studies may allow researchers a window into understanding how standardized alcohol cues might influence drinking behavior and might help inform prevention and intervention strategies.

Overall, assessing reactivity to alcohol cues using this set of photographs may facilitate a greater 
understanding of the affective processes associated with alcohol use and allow for more targeted behavioral change interventions for alcohol-related problems. 


\section{References}

Anton, R. F. (1999). What is craving? Models and implications for treatment. Alcohol Research \& Health: The Journal of the National Institute on Alcohol Abuse and Alcoholism, 23(3), 165-173. PMID: 10890811

Babor, T. F., Higgins-Biddle, J. C., Saunders, J. B., Monteiro, M. G. (2001). The Alcohol Use Disorders Identification Test: Guidelines for Use in Primary Care, Second Edition. District of Columbia, US: World Health Organization Press.

Billieux, J., Khazaal, Y., Oliveira, S., de Timary, P., Edel, Y., Zebouni, F., . . Van, d. L. (2011). The geneva appetitive alcohol pictures (GAAP): Development and preliminary validation. European Addiction Research, 17(5), 225-230. doi:10.1159/000328046

Bradley, M. M., \& Lang, P. J. (1994). Measuring emotion: The self-assessment manikin and the semantic differential. Journal of Behavior Therapy and Experimental Psychiatry, 25(1), 49-59. doi: 10.1016/0005-7916(94)90063-9

Buhrmester, M., Kwang, T., \& Gosling, S. D. (2011). Amazon's Mechanical Turk: A new source of inexpensive, yet high-quality, data?. Perspectives On Psychological Science,6(1), 3-5. doi:10.1177/1745691610393980

Carter, A. C. (2010). Examining the interface between alcohol expectancies, psychophysiological reactivity to alcohol picture cues, and risk for substance use disorders. ProQuest Information \& Learning). Dissertation Abstracts International: Section B: The Sciences and Engineering, 71(11)

Carter, B. L., \& Tiffany, S. T. (1999). Meta-analysis of cue-reactivity in addiction research. Addiction, 94(3), 327-340. doi:10.1046/j.1360-0443.1999.9433273.x

Carter, B., Robinson, J., Lam, C., Wetter, D., Tsan, J., Day, S., \& Cinciripini, P. (2006). A psychometric evaluation of cigarette stimuli used in a cue reactivity study. Nicotine \& Tobacco Research, 8(3), 361-369. doi: 10.1080/14622200600670215

Carver, C. S., \& White, T. L. (1994). Behavioral inhibition, behavioral activation, and affective responses to impending reward and punishment: The BIS/BAS scales. Journal of Personality and Social Psychology, 67(2), 319-333. doi:10.1037/0022-3514.67.2.319

Chou, S. P., Grant, B. F., \& Dawson, D. A. (1998). Alcoholic beverage preference and risks of alcohol-related medical consequences: A preliminary report from the national longitudinal alcohol epidemiologic survey. Alcoholism, Clinical and Experimental Research, 22(7), 1450-1455. doi: 10.1111/j.1530-0277.1998.tb03934.x

Cinciripini, P. M., Robinson, J. D., Carter, B. L., Lam, C., Wu, X., de Moor, C.,A., .. . Wetter, D. W. (2006). The effects of smoking deprivation and nicotine administration on emotional reactivity. Nicotine \& Tobacco Research: Official Journal of the Society for Research on Nicotine and Tobacco, 8(3), 379-392. doi:10.1080/14622200600670272 
Coffey, S. F., Saladin, M. E., Libet, J. M., Drobes, D. J., \& Dansky, B. S. (1999). Differential urge and salivary responsivity to alcohol cues in alcohol-dependent patients: A comparison of traditional and stringent classification approaches. Experimental and Clinical Psychopharmacology, 7(4), 464-472. doi:10.1037/1064-1297.7.4.464

Connolly, K. M., Coffey, S. F., Baschnagel, J. S., Drobes, D. J., \& Saladin, M. E. (2009). Evaluation of the alcohol craving questionnaire-now factor structures: Application of a cue reactivity paradigm. Drug and Alcohol Dependence, 103(1-2), 84-91. doi:10.1016/j.drugalcdep.2009.03.019

Cook, E. W., Melamed, B.G., Cuthbert, B.N., McNeil, D.W. \& Lang, P.J. (1988). Emotional imagery and the differential diagnosis of anxiety. Journal of Consulting and Clinical Psychology, 56, 734-740.

Cooney, N. L., Litt, M. D., Morse, P. A., Bauer, L. O., \& Gaupp, L. (1997). Alcohol cue reactivity, negative-mood reactivity, and relapse in treated alcoholic men. Journal of Abnormal Psychology, 106(2), 243-250. doi: 10.1037/0021-843X.106.2.243

Cox, W. M., \& Klinger, E. (1988). A motivational model of alcohol use. Journal of Abnormal Psychology, 97(2), 168-180. doi: 10.1037/0021-843X.97.2.168

Curtin, J., Barnett, N., Colby, S., Rohsenow, D., \& Monti, P. (2005). Cue reactivity in adolescents: measurement of separate approach and avoidance reactions. Journal of Studies On Alcohol, 66(3), 332-343. PMID:16047522

Drobes, D. J. (2002). Concurrent Alcohol and Tobacco Dependence. Alcohol Research \& Health, 26(2), 136-142. Retrieved from http://pubs.niaaa.nih.gov/publications/arh262/136-142.pdfDrobes, D. J., Carter, A. C., \& Goldman, M. S. (2009). Alcohol expectancies and reactivity to alcohol-related and affective cues. Experimental and Clinical Psychopharmacology, 17(1), 1-9. doi:10.1037/a0014482

Drummond, D., Tiffany, S. T., Glautier, S., \& Remington, B. (1995). Cue exposure in understanding and treating addictive behaviours. In D. Drummond, S. T. Tiffany, S. Glautier, B. Remington (Eds.), Addictive behaviour: Cue exposure theory and practice (pp. 1-17). Oxford England: John Wiley \& Sons.

Drummond, D. C., Litten, R. Z., Lowman, C., \& Hunt, W. A. (2000). Craving research: Future directions. Addiction (Abingdon, England), 95 Suppl 2, S247-S255. doi: 10.1046/j.13600443.95.8s2.13.x

Drummond, D. (2001). Theories of drug craving, ancient and modern. Addiction, 96(1), 33-46. doi: 10.1046/j.1360-0443.2001.961333.x

Franken, I.H., Muris, P. (2006). BIS/BAS personality characteristics and college students' substance use. Personality and Individual Differences, 40, 1497-1503. doi:10.1016/j.paid.2005.12.005 
Grüsser, ,S.M., Heinz, A., \& Flor, H. (2000). Standardized stimuli to assess drug craving and drug memory in addicts. Journal of Neural Transmission, 107(6), 715-720.

doi: $10.1007 / \mathrm{s} 007020070072$

Ham, L. S., Wang, Y., Kim, S. Y., \& Zamboanga, B. L. (2013). Measurement equivalence of the brief comprehensive effects of alcohol scale in a multiethnic sample of college students. Journal of Clinical Psychology, 69(4), 341-363. doi:10.1002/jclp.21904

Hays, R.D., Merz, J.F., Nicohlas, R. (1995). Response burden, reliability and validity of the CAGE, Short MAST, and AUDIT alcohol screening measures. Behavior Research Methods, Instruments, \& Computers. 27(2), 277-280. doi:10.3758/BF03204745

Heatherton T.F., Kozlowski L.T., Frecker R.C., Fagerstrom K.O. (1991). The Fagerstrom test for nicotine dependence: a revision of the Fagerstrom tolerance questionnaire. British Journal of Addiction, 86, 1119-1127. doi:10.1111/j.1360-0443.1991.tb01879.x

Hingson, R. (2010). Focus on: college drinking and related problems: magnitude and prevention of college drinking and related problems. Alcohol Research \& Health, 33(1), 45-54. doi: $10.1002 / 9780470686836$

Horn, J. L. (1965). A rationale and test for the number of factors in factor analysis. Psychometrika, 30(2), 179-185. doi:10.1007/BF02289447

Kambouropoulos, N., \& Staiger, P. K. (2001). The influence of sensitivity to reward on reactivity to alcohol-related cues. Addiction , 96(8), 1175-1185. doi: 10.1046/j.13600443.2001.968117510.x

Kassel, J. D., \& Shiffman, S. (1992). What can hunger teach us about drug craving? A comparative analysis of the two constructs. Advances in Behaviour Research \& Therapy, 14(3), 141-167. doi:10.1016/0146-6402(92)90006-A

Kidorf, M., Lang, A., \& Pelham, W. (1990). Beverage preference, beverage type and subject gender as determinants of alcohol consumption in the laboratory. Journal Of Studies On Alcohol, 51(4), 331-335. PMID: 2359306

Kramer, J. R., Chan, G., Hesselbrock, V. M., Kuperman, S., Bucholz, K. K., Edenberg, H. J., . . . Porjesz, B. (2010). A principal components analysis of the abbreviated desires for alcohol questionnaire (DAQ). Journal of Studies on Alcohol and Drugs, 71(1), 150-155. PMID: 20105425

Lang, P.J., Bradley, M.M., \& Cuthbert, B.N. (2008). International affective picture system (IAPS): Affective ratings of pictures and instruction manual. Technical report A-8. University of florida, gainesville, FL.

Lang, P. J., Bradley, M. M., \& Cuthbert, B. N. (1998). Emotion, motivation, and anxiety: Brain mechanisms and psychophysiology. Biological Psychiatry, 44(12), 1248-1263. doi: 10.1016/S0006-3223(98)00275-3 
Lee, E., Namkoong, K., Lee, C. H., An, S. K., \& Lee, B. O. (2006). Differences of photographs inducing craving between alcoholics and non-alcoholics. Yonsei Medical Journal, 47(4), 491-497. doi: 10.3349/ymj.2006.47.4.491

Lindman, R., \& Lang, A. R. (1986). Anticipated effects of alcohol consumption as a function of beverage type: A cross-cultural replication. International Journal Of Psychology, 21(6), 671-678. doi:10.1080/00207598608247613

Longman, R. S., Cota, A. A., Holden, R. R., \& Fekken, G. C. (1989). A regression equation for the parallel analysis criterion in principal components analysis: Mean and $95^{\text {th }}$ percentile eigenvalues. Multivariate Behavioral Research, 24, 59-69. doi:10.1207/s15327906mbr2401_4

Love, A., James, D., \& Willner, P. (1998). A comparison of two alcohol craving questionnaires. Addiction (Abingdon, England), 93(7), 1091-1102. doi:10.1046/j.13600443.1998.937109113.x

Mason, B. J., Light, J. M., Escher, T., \& Drobes, D. J. (2008). Effect of positive and negative affective stimuli and beverage cues on measures of craving in non treatment-seeking alcoholics. Psychopharmacology, 200(1), 141-150. doi:10.1007/s00213-008-1192-x

Mokdad, A. H., Marks, J. S., Stroup, D. F., \& Gerberding, J. L. (2000). Actual causes of death in the united states, 2000. JAMA: The Journal of the American Medical Association, 291(10), 1238-1245. doi: 10.1001/jama.291.10.1238.

Monti, P. M., Rohsenow, D. J., \& Hutchison, K. E. (2000). Toward bridging the gap between biological, psychobiological and psychosocial models of alcohol craving. Addiction (Abingdon, England), 95 Suppl 2, S229-S236. doi: 10.1046/j.1360-0443.95.8s2.11.x

Payne, T. J., Rychtarik, R. G., Rappaport, N. B., Smith, P. O., Etscheidt, M., Brown, T. A., \& Johnson, C. A. (1992). Reactivity to alcohol-relevant beverage and imaginal cues in alcoholics. Addictive Behaviors, 17(3), 209-217. doi:10.1016/0306-4603(92)90026-R

Pomerleau, O. F., Fertig, J., Baker, L., \& Cooney, N. (1983). Reactivity to alcohol cues in alcoholics and non-alcoholics: Implications for a stimulus control analysis of drinking. Addictive Behaviors, 8(1), 1-10. doi:10.1016/0306-4603(83)90048-5

Pulido, C., Brown, S. A., Cummins, K., Paulus, M. P., \& Tapert, S. F. (2010). Alcohol cue reactivity task development. Addictive Behaviors, 35(2), 84-90. doi:10.1016/j.addbeh.2009.09.006

Reynolds, E. K., \& Monti, P. M. (2013). The cue reactivity paradigm in addiction research. In J. MacKillop, H. de Wit (Eds.), The Wiley-Blackwell handbook of addiction psychopharmacology (pp. 381-410). Wiley-Blackwell. doi:10.1002/9781118384404.ch14

Rock, A. J., \& Kambouropoulos, N. (2012). The phenomenology of alcohol cue-reactivity: A partial replication and extension. Imagination, Cognition and Personality, 32(1), 75-93. doi:10.2190/IC.32.1.f 
Saladin, M., Drobes, D., Coffey, S., \& Libet, J. (2002). The human startle reflex and alcohol cue reactivity: effects of early versus late abstinence. Psychology Of Addictive Behaviors: Journal Of The Society Of Psychologists In Addictive Behaviors, 16(2), 98-105. doi: 10.1037//0893-164X.16.2.98

Schlauch, R. C., Gwynn-Shapiro, D., Stasiewicz, P. R., Molnar, D. S., \& Lang, A. R. (2013). Affect and craving: Positive and negative affect are differentially associated with approach and avoidance inclinations. Addictive Behaviors, 38(4), 1970-1979. doi:10.1016/j.addbeh.2012.12.003

Smits, D. J. M., \& Boeck, P. D. (2006). From BIS/BAS to the big five. European Journal of Personality, 20(4), 255-270. doi:10.1002/per.583

Spector, P.E. (1992). Summated rating scale construction: an introduction. In M.S. Lewis-Black (Series Ed.), Sage University Paper series on Quantitative Applications in the Social Sciences, No. 07-082, Newbury Park, CA: Sage.

Stasiewicz, P. R., Gulliver, S. B., Bradizza, C. M., Rohsenow, D. J., Torrisi, R., \& Monti, P. M. (1997). Exposure to negative emotional cues and alcohol cue reactivity with alcoholics: A preliminary investigation. Behaviour Research and Therapy, 35(12), 1143-1149. doi: 10.1016/S0005-7967(97)00047-8

Stritzke, W. G. K., Breiner, M. J., Curtin, J. J., \& Lang, A. R. (2004). Assessment of substance cue reactivity: Advances in reliability, specificity, and validity. Psychology of Addictive Behaviors: Journal of the Society of Psychologists in Addictive Behaviors, 18(2), 148159. doi:10.1037/0893-164X.18.2.148

Substance Abuse and Mental Health Services Administration [SAMHSA]. (2013). Results from the 2012 national survey on drug use and health: Summary of national findings (office of applied studies, NSDUH series H-46 HHS publication no. SMA 13-4795). Rockville, MD.

Vollstädt-Klein, S., Loeber, S., von der Goltz, G., Mann, K., \& Kiefer, F. (2009). Avoidance of alcohol-related stimuli increases during the early stage of abstinence in alcoholdependent patients. Alcohol and Alcoholism (Oxford, Oxfordshire), 44(5), 458-463. doi:10.1093/alcalc/agp056

Wallace, J., Malterer, M., \& Newman, J. (2009). Mapping gray's BIS and BAS constructs onto factor 1 and factor 2 of hare's psychopathy checklist - revised. Personality And Individual Differences, 47(8), 812-816. doi:10.1016/j.paid.2009.06.019

White, J.M., Staiger, P.K. (1991). Response to alcohol cues as a function of consumption level. Drug and Alcohol Dependence, 27(2), 191-195. doi:10.1016/0376-8716(91)09939-2

Wiers, R., Bartholow, B., van den Wildenberg, E., Thush, C., Engels, R., Sher, K., \& ... Stacy, A. (2007). Automatic and controlled processes and the development of addictive behaviors in adolescents: a review and a model. Pharmacology, Biochemistry, And Behavior, 86(2), 263-283. doi: 10.1016/j.pbb.2006.09.021 
Wrase, J., Grüsser, ,S.M., Klein, S., Diener, C., Hermann, D., Flor, H., . . Heinz, A. (2002). Development of alcohol-associated cues and cue-induced brain activation in alcoholics. European Psychiatry: The Journal of the Association of European Psychiatrists, 17(5), 287-291. doi:10.1016/S0924-9338(02)00676-4

Yen, J., Ko, C., Yen, C., Chen, C., \& Chen, C. (2009). The association between harmful alcohol use and Internet addiction among college students: comparison of personality. Psychiatry And Clinical Neurosciences, 63(2), 218-224. doi:10.1111/j.1440-1819.2009.01943.x 


\section{Appendix A}

Table 1

Demographic Summary

\begin{tabular}{|c|c|c|c|}
\hline \multirow[t]{2}{*}{ Gender } & Male & $74.4 \%$ & $n=145$ \\
\hline & Female & $25.6 \%$ & $n=50$ \\
\hline \multirow{8}{*}{ Race/Ethnicity } & Asian, Asian American, or Pacific & & \\
\hline & Islander & $56.4 \%$ & $n=110$ \\
\hline & White (Caucasian/non-Hispanic) & $34.9 \%$ & $n=68$ \\
\hline & Other & $4.6 \%$ & $n=9$ \\
\hline & Hispanic/Latino (Black) & $1.0 \%$ & $n=2$ \\
\hline & Native American / Alaskan Native & $1.5 \%$ & $n=3$ \\
\hline & Hispanic/Latino (White) & $1.0 \%$ & $n=2$ \\
\hline & $\begin{array}{l}\text { African American / Black } \\
\text { (non-Hispanic) }\end{array}$ & $.5 \%$ & $n=1$ \\
\hline \multirow[t]{5}{*}{ Age } & $20-29$ & $54.9 \%$ & $n=107$ \\
\hline & $30-39$ & $26.1 \%$ & $n=51$ \\
\hline & $40-49$ & $9.7 \%$ & $n=19$ \\
\hline & $50-59$ & $6.2 \%$ & $n=12$ \\
\hline & $60-69$ & $3.1 \%$ & $n=6$ \\
\hline \multirow[t]{7}{*}{ Education } & High School Degree or Less & $6.7 \%$ & $n=13$ \\
\hline & Some College & $15.9 \%$ & $n=31$ \\
\hline & Two Year College Degree & $3.6 \%$ & $n=7$ \\
\hline & Four Year College Degree & $28.2 \%$ & $n=55$ \\
\hline & Some Graduate Training & $4.6 \%$ & $n=9$ \\
\hline & Graduate Degree & $31.3 \%$ & $n=61$ \\
\hline & Professional Degree & $9.7 \%$ & $n=19$ \\
\hline \multirow[t]{2}{*}{ Employment } & Currently Employed & $82.1 \%$ & $n=160$ \\
\hline & Not Currently Employed & $17.9 \%$ & $n=35$ \\
\hline \multirow[t]{2}{*}{ Handedness } & Right & $93.3 \%$ & $n=182$ \\
\hline & Left & $6.7 \%$ & $n=13$ \\
\hline \multirow[t]{2}{*}{ College Student } & Yes & $9.7 \%$ & $n=19$ \\
\hline & No & $90.3 \%$ & $n=176$ \\
\hline
\end{tabular}


Living Conditions

Marital Status

Sexual Orientation

Region of Residence

Drink Type Preference
Alone

with Spouse/Partner

with Parents/Family

with Roommates

Single/Never Married

Married

Divorced

Less than $\$ 19,000$

$\$ 20,000-\$ 34,000$

$\$ 35,000-\$ 69,000$

More than $\$ 70,000$

Heterosexual

Homosexual

Bisexual

Midwest

Northeast

South

West

Beer

Wine

Hard Liquor

Mixed Drink

Fortified Wine

Wine Cooler

Liqueurs

Champagne
$8.2 \% \quad n=16$

$52.8 \% \quad n=103$

$28.7 \% \quad n=56$

$10.3 \% \quad n=20$

$44.6 \% \quad n=87$

$51.8 \% \quad n=101$

$3.6 \% \quad n=7$

$35.9 \% \quad n=70$

$33.8 \% \quad n=66$

$20.0 \% \quad n=39$

$10.3 \% \quad n=20$

$77.4 \% \quad n=151$

$3.1 \% \quad n=6$

$19.5 \% \quad n=38$

$28.7 \% \quad n=56$

$12.3 \% \quad n=24$

$43.1 \% \quad n=84$

$13.3 \% \quad n=26$

$37.4 \% \quad n=73$

$11.3 \% \quad n=22$

$13.3 \% \quad n=26$

$6.7 \% \quad n=13$

$3.1 \% \quad n=6$

$6.7 \% \quad n=13$

$6.2 \% \quad n=12$

$15.4 \% \quad n=30$ 
Table 2

Principle Components Analysis of Alcohol Cues: Obliquely Rotated Factor Loadings (Pattern Matrix) for Alcohol Craving Ratings $(N=195)$

Eigenvalues $\quad$ Factor 1

\begin{tabular}{|c|c|}
\hline Liquor,5,Pour & .95 \\
\hline MixedDrink,2,Bar & .94 \\
\hline Wine,3,Bar & .94 \\
\hline Liquor,2,Top & .94 \\
\hline Liquor,2,Bar & .94 \\
\hline MixedDrink,4,Top & .94 \\
\hline Liquor,4,Pour & .94 \\
\hline Wine, 1,Bar & .94 \\
\hline Wine,3,Pour & .93 \\
\hline Liquor,1,Bar & .93 \\
\hline MixedDrink,4,Pour & .93 \\
\hline MixedDrink,1,Top & .93 \\
\hline Beer,5,Top & .93 \\
\hline MixedDrink,5,Pour & .93 \\
\hline Liquor,4,Top & .93 \\
\hline Wine,2,Bar & .93 \\
\hline MixedDrink,1,Pour & .93 \\
\hline Liquor,2,Pour & .92 \\
\hline Wine,2,Pour & .92 \\
\hline MixedDrink,4,Bar & .92 \\
\hline Wine, 1, Top & .92 \\
\hline Wine,2,Top & .92 \\
\hline MixedDrink,5,Bar & .92 \\
\hline Beer,3,Top & .92 \\
\hline Liquor,1,Pour & .92 \\
\hline Liquor,4,Bar & .92 \\
\hline Wine,4,Pour & .92 \\
\hline Wine, 1,Pour & .92 \\
\hline MixedDrink,2,Top & .92 \\
\hline MixedDrink,1,Bar & .91 \\
\hline Liquor,3,Pour & .91 \\
\hline Wine,5,Bar & .91 \\
\hline Wine,3,Top & .91 \\
\hline MixedDrink,2,Pour & .91 \\
\hline Liquor,5,Bar & .91 \\
\hline
\end{tabular}




\begin{tabular}{ll} 
Beer,1,Bar & .91 \\
Liquor,3,Top & .91 \\
Liquor,1,Top & .91 \\
Wine,5,Top & .90 \\
MixedDrink,5,Top & .90 \\
Beer,5,Bar & .90 \\
Beer,4,Top & .90 \\
Liquor,5,Top & .90 \\
Beer,2,Top & .90 \\
Wine,4,Top & .89 \\
Liquor,3,Bar & .89 \\
Beer,2,Bar & .89 \\
MixedDrink,3,Top & .89 \\
Beer,2,Pour & .89 \\
Beer,1,Top & .89 \\
MixedDrink,3,Pour & .89 \\
Wine,5,Pour & .88 \\
Wine,4,Bar & .88 \\
Beer,4,Bar & .87 \\
Beer,4,Pour & .87 \\
Beer,3,Pour & .87 \\
MixedDrink,3,Bar & .86 \\
Beer,5,Pour & .86 \\
Beer,3,Bar & .86 \\
Beer,1,Pour & .82 \\
\hline
\end{tabular}

Note. Each cue description is listed in the following order: beverage type (Beer, Liquor, Mixed Drink, or Wine), which of 5 drink cues within the beverage type were presented (1, 2, 3, 4, or 5), beverage scene in photograph cue (Bar $=$ Behind Bar, Top $=$ Top of Bar, Pour $=$ Being Poured $)$. 
Table 3

Correlation Matrix

\begin{tabular}{|c|c|c|c|c|c|c|c|c|c|c|}
\hline & Mean & SD & Craving $^{\dagger}$ & DAQ & AUDIT & BIS & BASD & BASF & BASR & FTND \\
\hline Craving $^{\dagger}$ & 5.23 & 2.61 & .99 & & & & & & & \\
\hline DAQ & 20.54 & 9.35 & $.75^{* *}$ & .89 & & & & & & \\
\hline AUDIT & 12.79 & 8.17 & $.61^{* *}$ & $.61^{* *}$ & .87 & & & & & \\
\hline BIS & 19.28 & 3.54 & -.08 & -.025 & -.11 & .69 & & & & \\
\hline BASD & 11.50 & 2.51 & $.24^{* *}$ & $.28^{* * *}$ & .074 & .13 & .78 & & & \\
\hline BASF & 11.27 & 2.43 & $.26^{* *}$ & $.36^{* *}$ & $.15^{*}$ & -.001 & $.56^{* *}$ & .72 & & \\
\hline BASR & 16.08 & 3.05 & -.020 & .064 & $-.14^{*}$ & $.27^{* *}$ & $.64^{* *}$ & $.47^{* *}$ & .82 & \\
\hline FTND & 4.28 & 3.05 & .010 & $.21^{*}$ & $.19^{*}$ & .11 & .12 & .09 & .19 & .49 \\
\hline
\end{tabular}

Note. Chronbach's alphas are mentioned on the diagonal. Craving ${ }^{\dagger}=$ craving for alcohol following alcohol cues. DAQ = Desires for Alcohol Questionnaire, AUDIT = Alcohol Use Disorders Identification Test, BIS = Behavioral Inhibition System, BASD = Behavioral Activation System-Drive, BASF = Behavioral Activation System-Fun-seeking, BASR = Behavioral Activation Scale - Reward, FTND = Fagerström test of nicotine dependence, ${ }^{*} p<$ $.05,{ }^{* *} p<.01$. 
Table 4

Subjective Cue Ratings by Cue Type

\begin{tabular}{lccc}
\hline & Valence & Arousal & Craving \\
\hline Alcohol & $6.34^{*}(S D=1.52)$ & $5.42^{*}(S D=2.25)$ & $5.23^{*}(S D=2.61)$ \\
Neutral & $a=.99$ & $a=.99$ & $a=.99$ \\
& $4.53(S D=1.52)$ & $3.35 \quad(S D=1.59)$ & $2.84 \quad(S D=1.72)$ \\
Positive & $a=.93$ & $a=.94$ & $a=.95$ \\
& $6.73(S D=1.38)$ & $6.17 \quad(S D=1.84)$ & $4.59 \quad(S D=2.63)$ \\
Negative & $a=.91$ & $a=.94$ & $a=.97$ \\
& $3.24(S D=1.82)$ & $4.05 \quad(S D=2.10)$ & $2.48 \quad(S D=1.78)$ \\
& $a=.96$ & $a=.96$ & $a=.96$ \\
\hline
\end{tabular}

Note. Rating Scales ranged from 1 to 9 and were averaged within photo and rating type.

$*_{p}<.001$ (compared to the respective ratings following neutral cues) 
Table 5

Beverage Specific Subjective Cue Ratings by Cue Type

\begin{tabular}{|c|c|c|c|}
\hline & Valence & Arousal & Craving \\
\hline Beer & $\begin{array}{c}6.45 *(S D=1.58) \\
a=.97\end{array}$ & $\begin{array}{c}5.53 *(S D=2.33) \\
a=.99\end{array}$ & $\begin{array}{c}5.41 *(S D=2.67) \\
a=.99\end{array}$ \\
\hline Wine & $\begin{array}{c}6.33 \quad(S D=1.53) \\
a=.97\end{array}$ & $\begin{array}{c}5.40 \quad(S D=2.30) \\
a=.98\end{array}$ & $\begin{array}{c}5.18 \quad(S D=2.67) \\
a=.99\end{array}$ \\
\hline Mixed Drinks & $\begin{array}{c}6.32 \quad(S D=1.58) \\
a=.97\end{array}$ & $\begin{array}{c}5.38 \quad(S D=2.26) \\
a=.98\end{array}$ & $\begin{array}{c}5.20 \quad(S D=2.65) \\
a=.99\end{array}$ \\
\hline Hard Liquor & $\begin{array}{c}6.25 \quad(S D=1.60) \\
a=.97\end{array}$ & $\begin{array}{c}5.36 \quad(S D=2.27) \\
a=.98\end{array}$ & $\begin{array}{c}5.23 \quad(S D=2.65) \\
a=.99\end{array}$ \\
\hline
\end{tabular}

Note. Rating Scales ranged from 1 to 9 and were averaged within photo and rating type.

$* p<.001$ (compared to the respective ratings following neutral cues) 


\section{Appendix B}
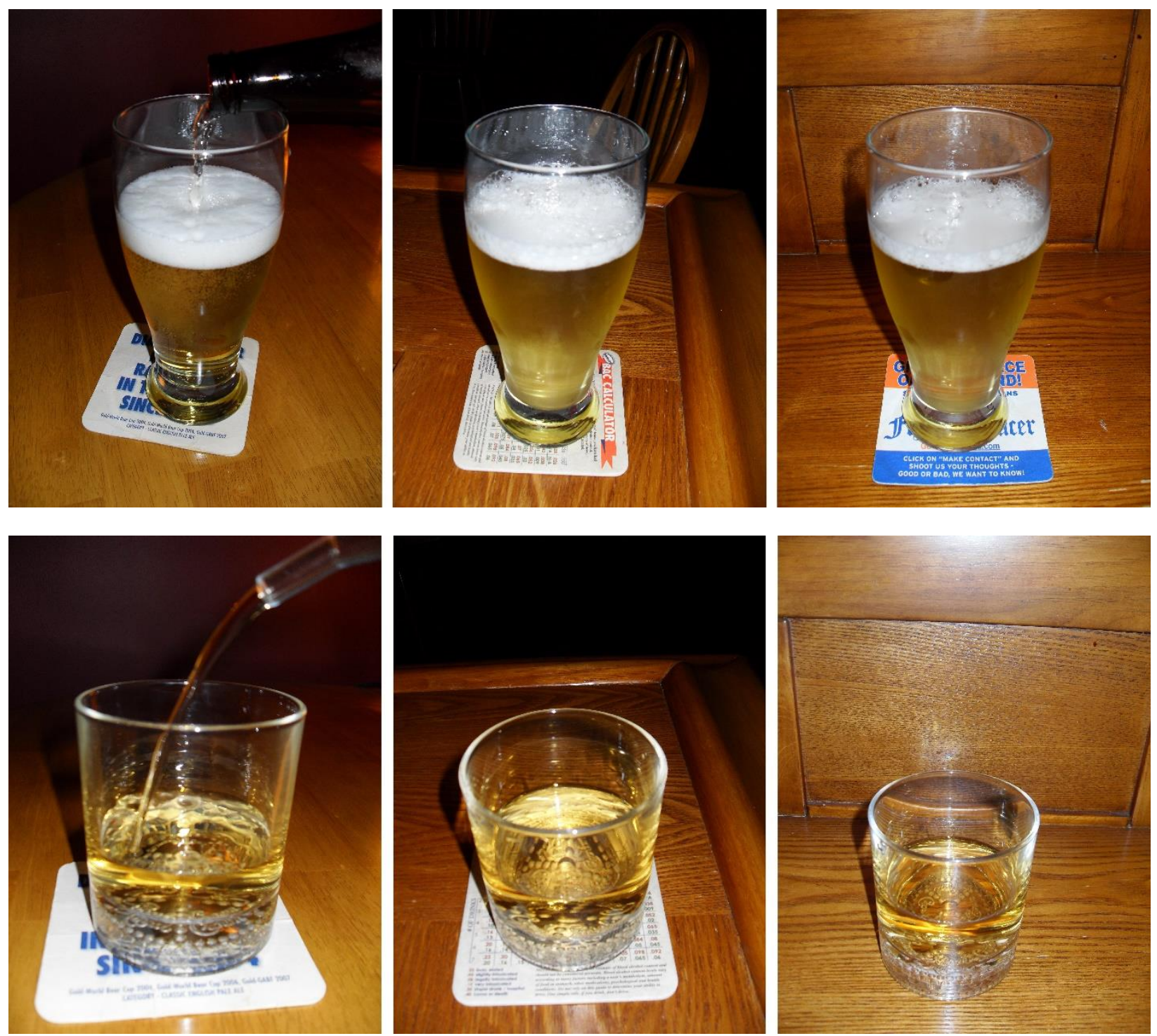

(Photos by author) 

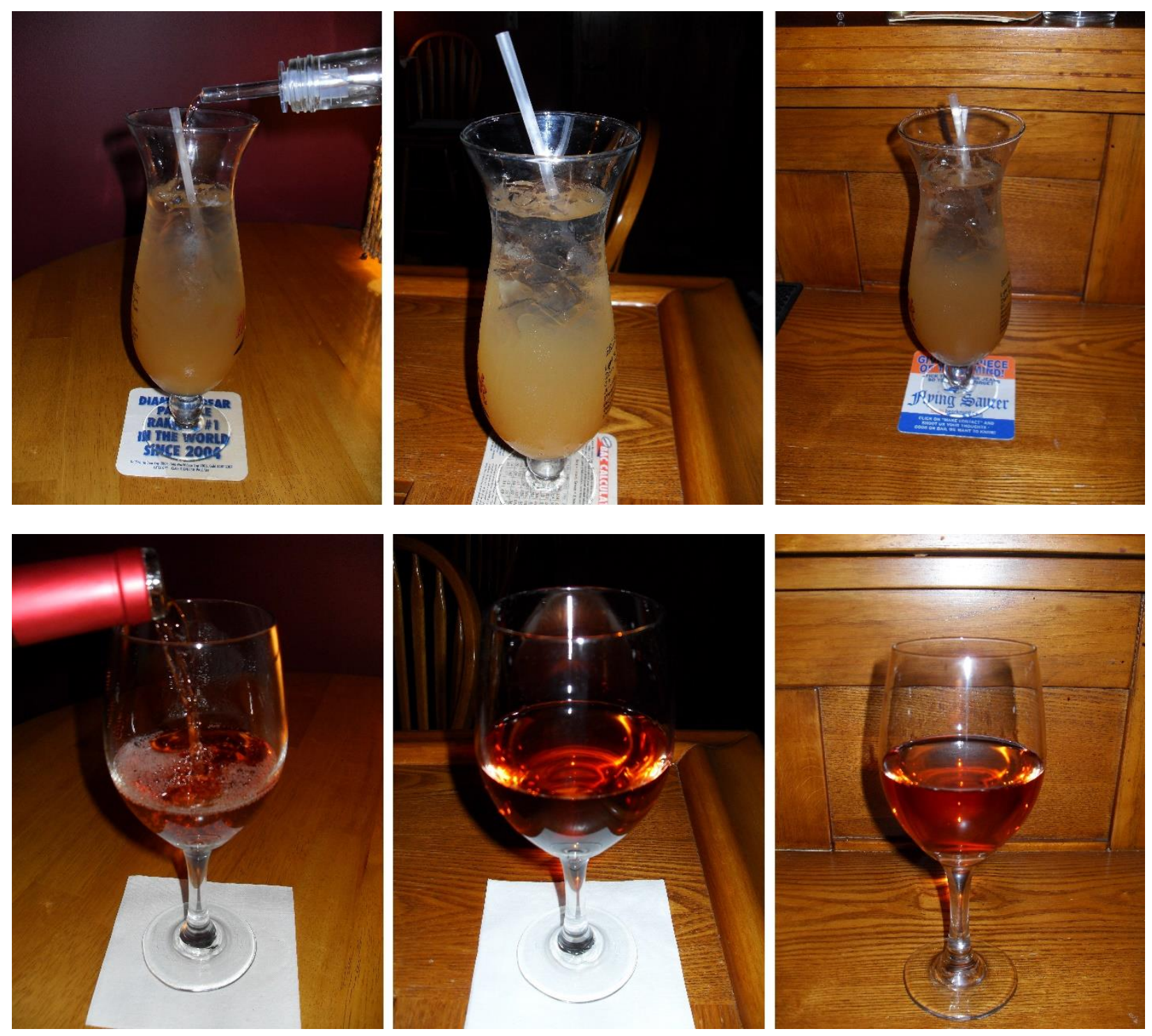

(Photos by author) 
August 16, 2013

Office of Research Compliance

Institutional Review Board

MEMORANDUM

TO:

David Lovett

Lindsay Ham

FROM:

Ro Windwalker

IRB Coordinator

RE:

PROJECT CONTINUATION \& MODIFICATION

IRB Protocol \#:

12-09-079

Protocol Title:

Alcohol and Responses to Pictures

Review Type:

$\bigotimes$ EXEMPT $\square$ EXPEDITED $\square$ FULL IRB

Previous Approval Period: $\quad$ Start Date: 09/11/2012 Expiration Date: 09/10/2013

New Expiration Date: $\quad$ 09/10/2014

Your request to extend and modify the referenced protocol has been approved by the IRB. If at the end of this period you wish to continue the project, you must submit a request using the form Continuing Review for IRB Approved Projects, prior to the expiration date. Failure to obtain approval for a continuation on or prior to this new expiration date will result in termination of the protocol and you will be required to submit a new protocol to the IRB before continuing the project. Data collected past the protocol expiration date may need to be eliminated from the dataset should you wish to publish. Only data collected under a currently approved protocol can be certified by the IRB for any purpose.

This protocol has been approved for 350 total participants. If you wish to make any modifications in the approved protocol, including enrolling more than this number, you must seek approval prior to implementing those changes. All modifications should be requested in writing (email is acceptable) and must provide sufficient detail to assess the impact of the change.

If you have questions or need any assistance from the IRB, please contact me at 210 Administration Building, 5-2208, or irb@uark.edu. 